\title{
CONDIÇÕES LMI PARA ESTABILIDADE ROBUSTA DE POLITOPOS DE MATRIZES POLINOMIAIS
}

\author{
Paulo J. de Oliveira* \\ jamesbk@uneb.br \\ Valter J. S. Leite \\ valterdiv.cefetmg.br \\ Ricardo C. L. F. Oliveira ${ }^{\dagger}$ \\ ricfowedt. fee.unicamp.br \\ Pedro L. D. Peres ${ }^{\dagger}$ \\ peres@dt.fee.unicamp.br \\ *DCET - UNEB, Av. Silveira Martins 2555, 41195-001 Salvador - BA - Brasil \\ ${ }^{\dagger}$ Faculdade de Engenharia Elétrica e de Computação, Universidade Estadual de Campinas \\ CP 6101, 13081-970, Campinas - SP - Brasil \\ ¥UnED Divinópolis - CEFET-MG, R. Monte Santo, 319, 35502-036, Divinópolis - MG - Brasil
}

\begin{abstract}
Sufficient conditions for checking the robust stability of a polytope of polynomial matrices are proposed in this paper. Simple feasibility tests performed in a convex set of linear matrix inequalities defined at the vertices of the polytope yield sufficient conditions for the robust stability of the entire domain. Both continuous-time (left half-plane) and discretetime stability (unit disk) are investigated. Improved sufficient conditions are also presented, containing the previous ones as special cases, providing an efficient numerical method for the robust stability analysis of polytopes of polynomial matrices. Numerical comparisons with quadratic stability and with results obtained from other recent methods in the literature show that the proposed conditions provide less conservative evaluations.
\end{abstract}

KEYWORDS: Robust stability, polynomial matrices, uncertain polynomials, linear matrix inequalities, parameter dependent Lyapunov functions.

Artigo submetido em 30/04/2002

1a. Revisão em 30/03/04; 2a. Revisão 24/07/04

Aceito sob recomendação do Ed. Assoc. Prof. Liu Hsu

\section{RESUMO}

Condições suficientes para a estabilidade robusta de um politopo de matrizes polinomiais são propostas neste artigo. Os testes de estabilidade são baseados em desigualdades matriciais lineares formuladas nos vértices do politopo de incertezas que, se factíveis, fornecem uma função de Lyapunov dependente de parâmetros que garante a estabilidade de qualquer polinômio matricial no domínio de incerteza. São analisados os casos de estabilidade a tempo contínuo (semi-plano esquerdo) e a tempo discreto (círculo unitário). Condições ainda mais abrangentes são também apresentadas, contendo as anteriores como casos particulares, proporcionando um método numérico eficaz para a análise da estabilidade robusta de polinômios matriciais. Experimentos numéricos mostram que as condições propostas produzem resultados menos conservadores quando comparados aos obtidos com a estabilidade quadrática e aos obtidos através de métodos recentes da literatura.

PALAVRAS-CHAVE: Estabilidade robusta, matrizes polinomiais, polinômios incertos, desigualdades matriciais lineares, funções de Lyapunov dependentes de parâmetros. 


\section{INTRODUÇÃO}

Matrizes polinomiais constituem um ferramental importante para o estudo de sistemas de controle. A dinâmica de muitos sistemas é descrita de maneira mais natural através de representações lineares fracionárias ou por matrizes cujos coeficientes são polinômios (Karl e Verghese, 1993), e várias abordagens de análise e de controle de sistemas se baseiam em matrizes polinomiais, como por exemplo, as chamadas abordagem polinomial (Kučera, 1979) e abordagem comportamental (Willems, 1991).

Em uma descrição por matrizes fracionárias, a matriz polinomial do denominador fornece informações sobre a dinâmica e a estabilidade do sistema (Kailath, 1980), e as especificações de desempenho estão associadas ao posicionamento dos zeros da matriz polinomial do denominador (Ackermann, 1993).

Na presença de parâmetros incertos, a análise da estabilidade robusta de sistemas dinâmicos passa pela verificação da estabilidade de matrizes polinomiais incertas, e uma das formas mais gerais de representação de incertezas paramétricas é a politópica (Barmish, 1994). Nas duas últimas décadas, inúmeros trabalhos sobre controle robusto de sistemas lineares incertos representados por modelos de estado e também por modelos freqüenciais têm surgido, e uma das metodologias mais empregadas é a baseada em funções de Lyapunov, como pode ser visto em Barmish (1994), Bhattacharyya et al. (1995) e Boyd et al. (1994). Uma boa discussão sobre a importância de matrizes polinomiais, a análise da estabilidade robusta de polinômios e a evolução dos métodos numéricos para a verificação das condições de estabilidade pode ser encontrada em Henrion, Arzelier, Peaucelle e Šebek (2001) e em Henrion, Bachelier e Šebek (2001). Para um estudo mais aprofundado sobre a natureza e propriedades gerais das matrizes polinomiais recomenda-se Gohberg et al. (1982).

Testes de estabilidade para polinômios têm tido destaque na literatura especializada nos últimos anos, como por exemplo o resultado de Kharitonov (Kharitonov, 1978) para polinômios com coeficientes definidos por intervalos, ou o chamado teorema das bordas (Bartlett et al., 1988) para politopos de polinômios. Veja também Barmish (1994) e Bhattacharyya et al. (1995) para maiores detalhes sobre estabilidade robusta de polinômios. Entretanto, a extensão desses resultados para tratar politopos de matrizes polinomiais não parece ser imediata, e testes conclusivos sobre a estabilidade de famílias de polinômios cujos coeficientes são funções multilineares dos parâmetros de incertezas são problemas NPdifíceis (Blondel e Tsitsiklis, 2000).

Condições formuladas em termos de desigualdades matriciais lineares (em inglês, LMIs - Linear Matrix Inequalities) recentemente publicadas na literatura para testar a estabi- lidade de um politopo de matrizes (Geromel et al., 1998), (de Oliveira et al., 1999), (Peaucelle et al., 2000) foram estendidas em Henrion, Arzelier, Peaucelle e Šebek (2001) para o caso de politopos de matrizes polinomiais. Mais recentemente, novas condições LMI ainda menos conservadoras foram publicadas para a análise de estabilidade de sistemas lineares discretos (Ramos e Peres, 2001a) e contínuos no tempo (Ramos e Peres, 2001b), (Ramos e Peres, 2002), (Leite et al., 2004). Em Leite e Peres (2003), condições mais abrangentes são apresentadas, também na forma de LMIs, contendo as anteriores como casos particulares e permitindo a análise de estabilidade em relação a uma região genérica do plano complexo.

Neste trabalho, condições suficientes para a estabilidade robusta de politopos de matrizes polinomiais são formuladas em termos de LMIs, obtidas a partir da extensão dos resultados de Ramos e Peres (2001a), Ramos e Peres (2001b), Ramos e Peres (2002). Essas condições produzem resultados menos conservadores que os obtidos com métodos baseados na estabilidade quadrática, e em geral, menos conservadores que os de Henrion, Arzelier, Peaucelle e Šebek (2001), principalmente quando a complexidade (isto é, grau, ordem e número de vértices) dos politopos de matrizes polinomiais aumenta, como mostram os testes realizados com polinômios matriciais estáveis na seção de exemplos. Finalmente, estendendo os resultados de Leite e Peres (2003) para a análise de estabilidade robusta de politopos de matrizes polinomiais, condições mais abrangentes que contêm as anteriores como casos particulares são apresentadas. Em todos os lemas e teoremas apresentados, a estabilidade robusta do politopo é assegurada por um simples teste de factibilidade de um conjunto de LMIs definidas nos vértices do domínio de incertezas, realizado de maneira eficiente por algoritmos de complexidade polinomial (Gahinet et al., 1995).

\section{PRELIMINARES}

Considere uma matriz polinomial quadrada $A(s)$ de dimensão $n$ e grau $d$ com coeficientes complexos que se escreve

$$
A(s)=A^{0}+A^{1} s+\cdots+A^{d} s^{d}
$$

Um zero de $A(s)$ pode ser definido (Kailath, 1980) como o valor complexo $\zeta$ para o qual ocorre uma perda de posto da matriz $A(s)$, ou seja, posto $A(\zeta)<$ posto $A(s)$, e pode ser determinado através do cômputo do determinante de $A(s)$.

Definida uma subregião $\mathcal{D}$ do plano complexo, o problema de análise da $\mathcal{D}$-estabilidade de $A(s)$ resume-se a determinar condições para que os zeros de $A(s)$ pertençam a $\mathcal{D}$.

Dadas $N$ matrizes polinomiais de coeficientes complexos $A_{1}(s), \ldots, A_{N}(s)$ de dimensão $n$ e grau $d$, define-se o politopo $\mathfrak{P}$ como sendo o conjunto de todas as matrizes polinomiais 
que se escrevem como

$$
A(s, \lambda)=\lambda_{1} A_{1}(s)+\cdots+\lambda_{N} A_{N}(s) ; \lambda_{j} \geq 0, \sum_{j=1}^{N} \lambda_{j}=1
$$

O objetivo deste trabalho é estabelecer condições que garantam que o politopo $\mathfrak{P}$ seja $\mathcal{D}$-estável, isto é, que todas as matrizes polinomiais $A(s, \lambda)$ sejam $\mathcal{D}$-estáveis. Uma descrição bastante abrangente para domínios de estabilidade $\mathcal{D}$ é dada por

$$
\mathcal{D}=\left\{s \in \mathbb{C}:\left[\begin{array}{l}
1 \\
s
\end{array}\right]^{*} B\left[\begin{array}{l}
1 \\
s
\end{array}\right]<0\right\}
$$

com $[\cdot]^{*}$ representando o conjugado transposto de $[\cdot]$. A escolha da matriz $B=B^{*} \in \mathbb{C}^{2}$ define a região a ser estudada. Neste trabalho, as regiões consideradas na seção de exemplos são o semi-plano esquerdo (análise de estabilidade de sistemas contínuos no tempo) e o círculo unitário (estabilidade de sistemas discretos no tempo), descritas respectivamente pelas matrizes

$$
B_{c}=\left[\begin{array}{ll}
0 & 1 \\
1 & 0
\end{array}\right] \quad ; \quad B_{d}=\left[\begin{array}{cc}
-1 & 0 \\
0 & 1
\end{array}\right]
$$

Para detalhes referentes a outras formas de definição de regiões do plano complexo, veja Chilali e Gahinet (1996), Henrion, Arzelier, Peaucelle e Šebek (2001), Henrion, Bachelier e Šebek (2001), Peaucelle et al. (2000). Por simplicidade, e sem perda de generalidade (Henrion, Bachelier e Šebek, 2001), assume-se que não há no politopo $\mathfrak{P}$ matrizes polinomiais com zeros no infinito, e também que o termo de grau $d$ é uma matriz identidade de dimensão $n$ denotada por $\mathbf{I}_{n}$.

Antes de passar aos resultados de estabilidade de matrizes polinomiais e às condições de estabilidade robusta propostas neste trabalho, defina a matriz constante $\mathcal{A}_{j} \in \mathbb{C}^{n \times(d+1) n}$ como

$$
\mathcal{A}_{j}=\left[\begin{array}{llll}
A_{j}^{0} & A_{j}^{1} & \cdots & A_{j}^{d}
\end{array}\right], A_{j}^{d}=\mathbf{I}_{n}
$$

contendo as matrizes coeficientes associadas com a matriz polinomial

$$
A_{j}(s)=A_{j}^{0}+A_{j}^{1} s+\cdots+\mathbf{I}_{n} s^{d}
$$

de dimensão $n$ e grau $d$. Denotando por $\mathcal{N}_{\mathcal{A}_{j}} \in \mathbb{C}^{(d+1) n \times d n}$ uma base do espaço nulo à direita da matriz $\mathcal{A}_{j}$, ou seja, $\mathcal{A}_{j} \mathcal{N}_{\mathcal{A}_{j}}=0$, e lembrando que, por hipótese, $A^{d}=\mathbf{I}_{n}$, tem-se

$$
\mathcal{N}_{A_{j}}=\left[\begin{array}{cccc}
\mathbf{I}_{n} & 0 & \cdots & 0 \\
0 & \mathbf{I}_{n} & \cdots & 0 \\
\vdots & \vdots & \ddots & \vdots \\
0 & 0 & \cdots & \mathbf{I}_{n} \\
-A_{j}^{0} & -A_{j}^{1} & \cdots & -A_{j}^{d-1}
\end{array}\right]
$$

Note que, utilizando a estrutura acima para a matriz $\mathcal{N}_{\mathcal{A}_{j}}$, tem-se que $\mathcal{N}_{\mathcal{A}(\lambda)}$ é uma base para o espaço nulo à direita de $\mathcal{A}(\lambda)$

$$
\mathcal{A}(\lambda)=\sum_{j=1}^{N} \lambda_{j} \mathcal{A}_{j} ; \lambda_{j} \geq 0, \sum_{j=1}^{N} \lambda_{j}=1
$$

que se escreve

$$
\mathcal{N}_{\mathcal{A}(\lambda)}=\sum_{j=1}^{N} \lambda_{j} \mathcal{N}_{\mathcal{A}_{j}} ; \lambda_{j} \geq 0, \sum_{j=1}^{N} \lambda_{j}=1
$$

Finalmente, a matriz de projeção $\mathcal{R} \in \mathbb{C}^{2 d n \times(d+1) n}$ é definida como

$$
\mathcal{R}=\left[\begin{array}{ccccc}
\mathbf{I}_{n} & 0 & \cdots & 0 & 0 \\
0 & \mathbf{I}_{n} & \cdots & 0 & 0 \\
\vdots & \vdots & \ddots & \vdots & \vdots \\
0 & 0 & \cdots & \mathbf{I}_{n} & 0 \\
0 & \mathbf{I}_{n} & 0 & \cdots & 0 \\
0 & 0 & \mathbf{I}_{n} & \cdots & 0 \\
\vdots & \vdots & \vdots & \ddots & \vdots \\
0 & 0 & 0 & \cdots & \mathbf{I}_{n}
\end{array}\right]
$$

e $\otimes$ representa o produto de Kronecker (Kailath, 1980).

\section{CONDIÇÕES PARA A ESTABILIDADE DO POLITOPO $\mathfrak{P}$}

Nesta seção, são apresentadas condições de estabilidade de matrizes polinomiais precisamente conhecidas, e condições de estabilidade robusta de politopos de matrizes polinomiais (estabilidade quadrática e uma condição suficiente de estabilidade robusta) sem especificar a região $\mathcal{D}$ investigada (deixando portanto em função da matriz $B$ definida em (3)), conforme apresentado em Henrion, Arzelier, Peaucelle e Šebek (2001). A partir desses resultados já conhecidos, serão enunciados os teoremas que constituem a principal contribuição deste trabalho.

LEMA 1 A matriz polinomial precisamente conhecida $A_{j}(s)$ é estável se e somente se existir uma matriz $P_{j} \in \mathbb{C}^{d n \times d n}$ solução do problema de factibilidade das LMIs

$$
\begin{gathered}
\mathcal{N}_{\mathcal{A}_{j}}^{*} \mathcal{R}^{*}\left(B \otimes P_{j}\right) \mathcal{R} \mathcal{N}_{\mathfrak{A}_{j}}<0 \\
P_{j}=P_{j}^{*}>0
\end{gathered}
$$

Prova: Veja Henrion, Bachelier e Šebek (2001).

LEMA 2 O politopo de matrizes polinomiais $\mathfrak{P}$ é robustamente estável se existirem matrizes $P_{j} \in \mathbb{C}^{d n \times d n}, j=1, \ldots, N$ e $Q \in \mathbb{C}^{2 d n \times n}$ solução do problema de factibilidade das LMIs

$$
\left[\begin{array}{l}
\mathcal{R} \\
\mathcal{A}_{j}
\end{array}\right]^{*}\left[\begin{array}{cc}
B \otimes P_{j} & Q \\
Q^{*} & 0
\end{array}\right]\left[\begin{array}{l}
\mathcal{R} \\
\mathcal{A}_{j}
\end{array}\right]<0 ; j=1, \ldots, N
$$




$$
P_{j}=P_{j}^{*}>0 \quad ; j=1, \ldots, N
$$

Prova: A prova completa pode ser vista em Henrion, Arzelier, Peaucelle e Šebek (2001). A idéia principal é explorar a existência da variável $Q$, que representa um grau de liberdade a mais no problema e que é comum às LMIs dadas na equação (13), para construir uma condição de estabilidade simultaneamente linear em $A_{j}$ e $P_{j}$. Note que (13) pode ser reescrita como

$$
\mathcal{R}^{*}\left(B \otimes P_{j}\right) \mathcal{R}+\mathcal{A}_{j}^{*} Q^{*} \mathcal{R}+\mathcal{R}^{*} Q \mathcal{A}_{j}<0
$$

que, multiplicada termo a termo por $\lambda_{j}$ e somada sobre todos os índices $j$, fornece

$$
\left[\begin{array}{c}
\mathcal{R} \\
\mathcal{A}(\lambda)
\end{array}\right]^{*}\left[\begin{array}{cc}
B \otimes P(\lambda) & Q \\
Q^{*} & 0
\end{array}\right]\left[\begin{array}{c}
\mathcal{R} \\
\mathcal{A}(\lambda)
\end{array}\right]<0
$$

$\operatorname{com} \mathcal{A}(\lambda)$ dada em (8) e

$$
P(\lambda)=P(\lambda)^{*}=\sum_{j=1}^{N} \lambda_{j} P_{j}>0, \lambda_{j} \geq 0, \sum_{j=1}^{N} \lambda_{j}=1
$$

A partir de (16), tem-se

$$
\begin{gathered}
\mathcal{N}_{\mathscr{A}(\lambda)}^{*}\left[\begin{array}{c}
\mathcal{R} \\
\mathcal{A}(\lambda)
\end{array}\right]^{*}\left[\begin{array}{cc}
B \otimes P(\lambda) & Q \\
Q^{*} & 0
\end{array}\right]\left[\begin{array}{c}
\mathcal{R} \\
\mathcal{A}(\lambda)
\end{array}\right] \mathcal{N}_{\mathcal{A}(\lambda)} \\
=\mathcal{N}_{\mathscr{A}(\lambda)}^{*} \mathcal{R}^{*}(B \otimes P(\lambda)) \mathcal{R} \mathcal{N}_{\mathcal{A}(\lambda)}<0
\end{gathered}
$$

Usando o resultado do Lema 1, conclui-se que a matriz polinomial $A(s, \lambda)$ dada em (2) é estável para todo $\lambda$ admissível e, portanto, o politopo $\mathfrak{P}$ é estável.

Uma condição mais conservadora para a estabilidade robusta do politopo $\mathfrak{P}$ é obtida quando se exige, no Lema 2 , que $P=P_{1}=P_{2}=\cdots=P_{N}$, resultando na condição enunciada no lema a seguir (estabilidade quadrática de politopos de matrizes polinomiais).

LEMA 3 O politopo de matrizes polinomiais $\mathfrak{P}$ é quadraticamente estável se e somente se existirem matrizes $P \in$ $\mathbb{C}^{d n \times d n}$ e $Q \in \mathbb{C}^{2 d n \times n}$ solução do problema de factibilidade das LMIs

$$
\begin{gathered}
{\left[\begin{array}{c}
\mathcal{R} \\
\mathcal{A}_{j}
\end{array}\right]^{*}\left[\begin{array}{cc}
B \otimes P & Q \\
Q^{*} & 0
\end{array}\right]\left[\begin{array}{c}
\mathcal{R} \\
\mathcal{A}_{j}
\end{array}\right]<0 ; j=1, \ldots, N} \\
P=P^{*}>0
\end{gathered}
$$

Prova: A partir dos resultados anteriores e por analogia com o conceito de estabilidade quadrática de sistemas lineares, a prova é imediata (Henrion, Arzelier, Peaucelle e Šebek, 2001).
Note que a estabilidade de um politopo de matrizes polinomiais descrito por $\mathcal{A}(\lambda)$ pode ser verificada através da expressão que está no lado esquerdo de (18), isto é, se para todo $\lambda$ considerado no domínio de incertezas existir uma matriz $P(\lambda)=P(\lambda)^{*}>0$ verificando

$$
\mathcal{N}_{\mathcal{A}(\lambda)}^{*} \mathcal{R}^{*}(B \otimes P(\lambda)) \mathcal{R} \mathcal{N}_{\mathcal{A}(\lambda)}<0
$$

então o politopo $\mathfrak{P}$ é estável.

Usando o Lema 1, pode-se analisar um ponto precisamente conhecido dentro do domínio e, com o Lema 3, constatase a estabilidade quadrática do politopo. O Lema 2 estabelece uma condição suficiente para que a combinação convexa das matrizes $P_{j}=P_{j}^{*}>0$ forneça uma matriz $P(\lambda)$ solução de (21). Através do Lema 2, a condição (21) pode ser testada sem a necessidade de uma discretização exaustiva do espaço de parâmetros $\lambda$, pois apenas a factibilidade das LMIs descritas nos $N$ vértices do politopo precisa ser verificada. Note também que, para as duas regiões de estabilidade descritas através das matrizes $B_{c}$ e $B_{d}$ em (4), a estabilidade quadrática de um politopo de matrizes polinomiais poderia ser investigada diretamente através da existência de uma mesma matriz $P=P^{*}>0$ satisfazendo a expressão (21) nos vértices do politopo, sem a necessidade da matriz $Q$ e da formulação aumentada do Lema 3. De fato, particionando a matriz $\mathcal{R} \mathcal{N}_{\mathcal{A}(\lambda)} \in \mathbb{C}^{2 d n \times d n}$ em dois blocos

$$
\mathcal{R} \mathcal{N}_{\mathcal{A}(\lambda)}=\left[\frac{\mathbf{I}_{d n}}{\Xi(\lambda)}\right]
$$

tem-se que $\Xi(\lambda) \in \mathbb{C}^{d n \times d n}$ é uma matriz na forma blococompanheira dada por

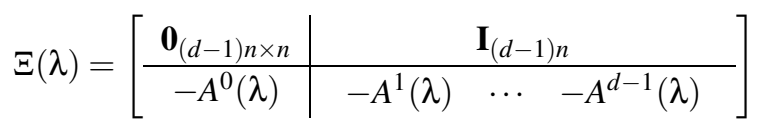

e, portanto, para $B=B_{c}$ e $P(\lambda)=P$, o lado esquerdo de (21) pode ser reescrito como

$$
\begin{gathered}
{\left[\mathbf{I}_{d n} \mid \Xi^{*}(\lambda)\right]\left(\left[\begin{array}{c|c}
0 & P \\
\hline P & 0
\end{array}\right]\right)\left[\begin{array}{c}
\mathbf{I}_{d n} \\
\hline \Xi(\lambda)
\end{array}\right]=} \\
=\Xi^{*}(\lambda) P+P \Xi(\lambda)
\end{gathered}
$$

A condição acima é definida negativa para todo $\lambda$ no politopo $\mathfrak{P}$ se e somente se

$$
\Xi_{j}^{*} P+P \Xi_{j}<0, j=1, \ldots, N
$$

com

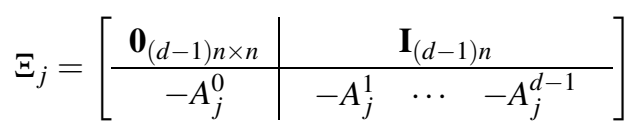


De maneira similar, para $B=B_{d}$, tem-se a estabilidade de $\mathfrak{P}$ para todo $\lambda$ se e somente se as expressões convexas são verificadas (veja Geromel et al. (1991) para detalhes)

$$
\Xi_{j}^{*} P \Xi_{j}-P<0, j=1, \ldots, N
$$

Os teoremas a seguir fornecem novas condições suficientes para que $P(\lambda)$ dado por (17) satisfaça (21). O resultado é uma extensão de condições recentemente publicadas para análise de estabilidade robusta de politopos de matrizes (Ramos e Peres, 2001a), (Ramos e Peres, 2001b), (Ramos e Peres, 2002).

TEOREMA 1 (caso contínuo): O politopo de matrizes polinomiais $\mathfrak{P}$ é robustamente estável se existirem matrizes $P_{j} \in \mathbb{C}^{d n \times d n}, j=1, \ldots, N$, solução do problema de factibilidade das LMIs

$$
\begin{gathered}
\mathcal{N}_{\mathcal{A}_{j}}^{*} \mathcal{R}^{*}\left(B_{c} \otimes P_{j}\right) \mathcal{R} \mathcal{N}_{\mathfrak{A}_{j}}<-\mathbf{I} ; j=1, \ldots, N \\
\mathcal{N}_{\mathscr{A}_{j}}^{*} \mathcal{R}^{*}\left(B_{c} \otimes P_{k}\right) \mathcal{R} \mathcal{N}_{\mathcal{A}_{j}}+\mathcal{N}_{\mathscr{A}_{k}}^{*} \mathcal{R}^{*}\left(B_{c} \otimes P_{j}\right) \mathcal{R} \mathcal{N}_{\mathfrak{A}_{k}} \\
<\frac{2}{N-1} \mathbf{I} ; j=1, \ldots, N-1 ; k=j+1, \ldots, N \\
P_{j}=P_{j}^{*}>0 ; j=1, \ldots, N
\end{gathered}
$$

com $B_{c}, \mathcal{N}_{\mathcal{A}_{j}}$ e $\mathcal{R}$ dados, respectivamente, em (4), (7) e (10). No caso afirmativo, $P(\lambda)$ dada por (17) é definida positiva e satisfaz (21) para todo $\mathcal{A}(\lambda)$.

Prova: É claro que $P(\lambda)$ dada por (17) é definida positiva. Utilizando $A(\lambda)$ dado em (8) e desenvolvendo (18) com $B_{c}$, $\mathcal{N}_{\mathcal{A}(\lambda)}$ e $\mathcal{R}$ dados respectivamente em (4), (9) e (10), tem-se

$$
\begin{aligned}
& \mathcal{N}_{\mathcal{A}(\lambda)}^{*} \mathcal{R}^{*}\left(B_{c} \otimes P(\lambda)\right) \mathcal{R} \mathcal{N}_{\mathcal{A}(\lambda)}= \\
&=\sum_{j=1}^{N} \lambda_{j}^{2}\left(\mathcal{N}_{\mathcal{A}_{j}}^{*} \mathcal{R}^{*}\left(B_{c} \otimes P_{j}\right) \mathcal{R} \mathcal{N}_{\mathcal{A}_{j}}\right) \\
&+\sum_{j=1}^{N-1} \sum_{k=j+1}^{N} \lambda_{j} \lambda_{k}\left(\mathcal{N}_{\mathcal{A}_{j}}^{*} \mathcal{R}^{*}\left(B_{c} \otimes P_{k}\right) \mathcal{R} \mathcal{N}_{\mathcal{A}_{j}}\right. \\
&\left.+\mathcal{N}_{\mathcal{A}_{k}}^{*} \mathcal{R}^{*}\left(B_{c} \otimes P_{j}\right) \mathcal{R} \mathcal{N}_{\mathcal{A}_{k}}\right)
\end{aligned}
$$

Impondo as condições (22)-(23) do teorema (note que $\lambda_{j} \lambda_{k} \geq$ 0) tem-se

$$
\begin{aligned}
\mathcal{N}_{\mathcal{A}(\lambda)}^{*} \mathcal{R}^{*}\left(B_{c} \otimes P(\lambda)\right) \mathcal{R} \mathcal{N}_{\mathcal{A}(\lambda)}< \\
-\left(\sum_{j=1}^{N} \lambda_{j}^{2}-\sum_{j=1}^{N-1} \sum_{k=j+1}^{N} \lambda_{j} \lambda_{k} \frac{2}{N-1}\right) \mathbf{I} \leq 0
\end{aligned}
$$

pois a expressão dentro dos parênteses é sempre maior ou igual a zero. De fato,

$$
\begin{aligned}
0 \leq \sum_{j=1}^{N-1} \sum_{k=j+1}^{N}\left(\lambda_{j}-\lambda_{k}\right)^{2}= & \\
& =(N-1) \sum_{j=1}^{N} \lambda_{j}^{2}-2 \sum_{j=1}^{N-1} \sum_{k=j+1}^{N} \lambda_{j} \lambda_{k}
\end{aligned}
$$

Algumas observações podem ser feitas sobre o resultado do Teorema 1. Primeiramente, é importante observar que podese impor que o lado esquerdo da condição (22) seja menor que -I sem perda de generalidade, já que a expressão é homogênea em $P_{j}$ e a estabilidade dos vértices do politopo é condição necessária para a estabilidade do domínio. Essa folga é utilizada na condição (23), à qual se permite que seja até definida positiva. Note também que, para polinômios matriciais precisamente conhecidos $(N=1)$, apenas a condição (22) precisa ser testada para $P_{1}=P_{1}^{*}>0$, recaindo-se portanto no resultado do Lema 1. Além disso, se uma mesma matriz $P=P^{*}>0$ existe verificando as condições do Lema 3 (ou seja, o politopo é quadraticamente estável), então $P=$ $P_{1}=P_{2}=\cdots=P_{N}$ é uma solução factível para (22), o que implica que (23) é sempre verificada neste caso. Como conclusão, as condições do Teorema 1 englobam a estabilidade quadrática como um caso particular. Outra observação interessante é que a condição $\sum_{j=1}^{N} \alpha_{j}=1$ não é utilizada no Teorema 1 , bastando apenas que os parâmetros $\alpha_{j}$ sejam todos positivos e que $\sum_{j=1}^{N} \alpha_{j}>0$, indicando que, conhecendo-se os vértices $A_{j}$ do politopo, pode-se concluir sobre a estabilidade de qualquer combinação positiva $\sum_{j=1}^{N} \alpha_{j} A_{j}$.

Em termos de complexidade, métodos de resolução baseados em pontos interiores requerem um número de operações em ponto flutuante da ordem de $O\left(K^{3} L\right)$, sendo $K$ o número de variáveis escalares envolvidas no problema e $L$ o número de linhas das LMIs (Boyd et al., 1994), (Gahinet et al., 1995). Embora o número de variáveis escalares envolvidas no Teorema $1\left(K_{T 1}=N d n(d n+1) / 2\right)$ seja menor do que no Lema 2 $\left(K_{L 2}=N d n(d n+1) / 2+2 d n^{2}\right)$, o número de LMIs, dado por $L_{T 1}=N(N+3) / 2$, cresce com $N^{2}$ e esse fator torna-se preponderante para $N$ grande $(N>20)$. Finalmente, é interessante observar que, assim como o resultado do Lema 2 recai na análise de estabilidade robusta de sistemas com incerteza politópica (Peaucelle et al., 2000) para $A_{j}(s)=A_{j}-s \mathbf{I}_{n}$, $d=1$, as condições do Teorema 1 se reduzem ao resultado de Ramos e Peres (2001b), Ramos e Peres (2002). Nesse caso particular, tem-se

$$
\mathcal{R} \mathcal{N}_{A_{j}}=\left[\begin{array}{c}
\mathbf{I}_{n} \\
A_{j}
\end{array}\right]
$$


e, portanto, as condições (22)-(23) escrevem-se

$$
\begin{gathered}
{\left[\begin{array}{c}
\mathbf{I}_{n} \\
A_{j}
\end{array}\right]^{\prime}\left[\begin{array}{cc}
0 & P_{j} \\
P_{j} & 0
\end{array}\right]\left[\begin{array}{c}
\mathbf{I}_{n} \\
A_{j}
\end{array}\right]=} \\
=A_{j}^{\prime} P_{j}+P_{j} A_{j}<-\mathbf{I} ; j=1, \ldots, N \\
{\left[\begin{array}{c}
\mathbf{I}_{n} \\
A_{j}
\end{array}\right]^{\prime}\left[\begin{array}{cc}
0 & P_{k} \\
P_{k} & 0
\end{array}\right]\left[\begin{array}{c}
\mathbf{I}_{n} \\
A_{j}
\end{array}\right]+\left[\begin{array}{c}
\mathbf{I}_{n} \\
A_{k}
\end{array}\right]^{\prime}\left[\begin{array}{cc}
0 & P_{j} \\
P_{j} & 0
\end{array}\right]\left[\begin{array}{c}
\mathbf{I}_{n} \\
A_{k}
\end{array}\right]=} \\
=A_{j}^{\prime} P_{k}+P_{k} A_{j}+A_{k}^{\prime} P_{j}+P_{j} A_{k}<\frac{2}{N-1} \mathbf{I} ; \\
j=1, \ldots, N-1 ; k=j+1, \ldots, N
\end{gathered}
$$

TeOrema 2 (caso discreto): O politopo de matrizes polinomiais $\mathfrak{P}$ é robustamente estável se existirem matrizes $P_{j} \in \mathbb{C}^{d n \times d n}, j=1, \ldots, N$, soluções do problema de factibilidade das LMIs

$$
\begin{aligned}
& \mathcal{N}_{\mathscr{A}_{j}}^{*} \mathcal{R}^{*}\left(B_{d} \otimes P_{j}\right) \mathcal{R} \mathcal{N}_{\mathcal{A}_{j}}<-\mathbf{I} ; j=1, \ldots, N \\
& \mathcal{N}_{\mathscr{A}_{j}}^{*} \mathcal{R}^{*}\left(B_{d} \otimes P_{j}\right) \mathcal{R} \mathcal{N}_{A_{k}}+\mathcal{N}_{\mathscr{A}_{k}}^{*} \mathcal{R}^{*}\left(B_{d} \otimes P_{j}\right) \mathcal{R} \mathcal{N}_{\mathfrak{A}_{j}} \\
& +\mathcal{N}_{\mathscr{A}_{j}}^{*} \mathcal{R}^{*}\left(B_{d} \otimes P_{k}\right) \mathcal{R} \mathcal{N}_{\mathfrak{A}_{j}}<\frac{1}{(N-1)^{2}} \mathbf{I} ; \\
& j=1, \ldots, N, k \neq j, k=1, \ldots, N \\
& \mathcal{N}_{\mathcal{A}_{j}}^{*} \mathcal{R}^{*}\left(B_{d} \otimes P_{k}\right) \mathcal{R} \mathcal{N}_{\mathcal{A}_{\ell}}+\mathcal{N}_{\mathcal{A}_{\ell}}^{*} \mathcal{R}^{*}\left(B_{d} \otimes P_{k}\right) \mathcal{R} \mathcal{N}_{\mathcal{A}_{j}} \\
& +\mathcal{N}_{\mathscr{A}_{k}}^{*} \mathcal{R}^{*}\left(B_{d} \otimes P_{j}\right) \mathcal{R} \mathcal{N}_{\mathfrak{A}_{\ell}}+\mathcal{N}_{\mathfrak{A}_{\ell}}^{*} \mathcal{R}^{*}\left(B_{d} \otimes P_{j}\right) \mathcal{R} \mathcal{N}_{\mathfrak{A}_{k}} \\
& +\mathcal{N}_{\mathscr{A}_{j}}^{*} \mathcal{R}^{*}\left(B_{d} \otimes P_{\ell}\right) \mathcal{R} \mathcal{N}_{A_{k}}+\mathcal{N}_{\mathscr{A}_{k}}^{*} \mathcal{R}^{*}\left(B_{d} \otimes P_{\ell}\right) \mathcal{R} \mathcal{N}_{A_{j}}
\end{aligned}
$$

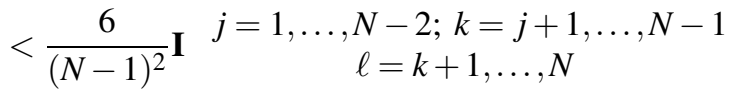

$$
\begin{aligned}
& P_{j}=P_{j}^{*}>0 \quad ; \quad j=1, \ldots, N
\end{aligned}
$$

com $B_{d}, \mathcal{N}_{\mathcal{A}_{j}}$ e $\mathcal{R}$ dados, respectivamente, em (4), (7) e (10). No caso afirmativo, $P(\lambda)$ dada por (17) é definida positiva e satisfaz (21) para todo $\mathcal{A}(\lambda)$.

Prova: Claramente, $P(\lambda)$ dada por (17) é definida positiva pois (31) é verificada. Utilizando $A(\lambda)$ dado em (8) e desenvolvendo (21) com $B=B_{d}, \mathcal{N}_{\mathcal{A}(\lambda)}$ e $\mathcal{R}$ dados respectivamente em (4), (9) e (10), tem-se

$$
\begin{aligned}
& \mathcal{N}_{\mathcal{A}(\lambda)}^{*} \mathcal{R}^{*}\left(B_{d} \otimes P(\lambda)\right) \mathcal{R} \mathcal{N}_{\mathcal{A}(\lambda)}= \\
& =\sum_{j=1}^{N} \lambda_{j}^{3}\left(\mathcal{N}_{\mathscr{A}_{j}}^{*} \mathcal{R}^{*}\left(B_{d} \otimes P_{j}\right) \mathcal{R} \mathcal{N}_{\mathcal{A}_{j}}\right) \\
& +\sum_{j=1}^{N} \sum_{k \neq j ; k=1}^{N} \lambda_{j}^{2} \lambda_{k}\left(\mathcal{N}_{\mathcal{A}_{j}}^{*} \mathcal{R}^{*}\left(B_{d} \otimes P_{j}\right) \mathcal{R} \mathcal{N}_{\mathcal{A}_{k}}\right. \\
& \left.+\mathcal{N}_{\mathscr{A}_{k}}^{*} \mathcal{R}^{*}\left(B_{d} \otimes P_{j}\right) \mathcal{R} \mathcal{N}_{\mathfrak{A}_{j}}+\mathcal{N}_{\mathscr{A}_{j}}^{*} \mathcal{R}^{*}\left(B_{d} \otimes P_{k}\right) \mathcal{R} \mathcal{N}_{\mathfrak{A}_{j}}\right) \\
& +\sum_{j=1}^{N-2} \sum_{k=j+1}^{N-1} \sum_{\ell=k+1}^{N} \lambda_{j} \lambda_{k} \lambda_{\ell}\left(\mathcal{N}_{\mathscr{A}_{j}}^{*} \mathcal{R}^{*}\left(B_{d} \otimes P_{k}\right) \mathcal{R} \mathcal{N}_{\mathcal{A}_{\ell}}\right. \\
& +\mathcal{N}_{\mathscr{A}_{\ell}}^{*} \mathcal{R}^{*}\left(B_{d} \otimes P_{k}\right) \mathcal{R} \mathcal{N}_{\mathcal{A}_{j}}+\mathcal{N}_{\mathcal{A}_{k}}^{*} \mathcal{R}^{*}\left(B_{d} \otimes P_{j}\right) \mathcal{R} \mathcal{N}_{\mathcal{A}_{\ell}} \\
& +\mathcal{N}_{\mathcal{A}_{\ell}}^{*} \mathcal{R}^{*}\left(B_{d} \otimes P_{j}\right) \mathcal{R} \mathcal{N}_{\mathcal{A}_{k}}+\mathcal{N}_{\mathcal{A}_{j}}^{*} \mathcal{R}^{*}\left(B_{d} \otimes P_{\ell}\right) \mathcal{R} \mathcal{N}_{A_{k}} \\
& \left.+\mathcal{N}_{\mathfrak{A}_{k}}^{*} \mathcal{R}^{*}\left(B_{d} \otimes P_{\ell}\right) \mathcal{R} \mathcal{N}_{\mathcal{A}_{j}}\right)
\end{aligned}
$$

Impondo as condições (28)-(30) e lembrando que $\lambda_{j} \geq 0, \forall j$ e $\sum_{j=1}^{N} \lambda_{j}=1$ tem-se

$$
\begin{aligned}
& \mathcal{N}_{\mathcal{A}(\lambda)}^{*} \mathcal{R}^{*}\left(B_{d} \otimes P(\lambda)\right) \mathcal{R} \mathcal{N}_{\mathcal{A}(\lambda)}< \\
&-\left(\sum_{j=1}^{N} \lambda_{j}^{3}-\frac{1}{(N-1)^{2}} \sum_{j=1}^{N} \sum_{k \neq j ; k=1}^{N} \lambda_{j}^{2} \lambda_{k}\right. \\
&\left.\quad-\frac{6}{(N-1)^{2}} \sum_{j=1}^{N-2} \sum_{k=j+1}^{N-1} \sum_{\ell=k+1}^{N} \lambda_{j} \lambda_{k} \lambda_{\ell}\right) \mathbf{I}
\end{aligned}
$$

Para analisar o sinal da expressão dentro dos parênteses acima, usando o resultado de Ramos e Peres (2001a), defina

$$
\begin{gathered}
0 \leq \Theta \triangleq \sum_{j=1}^{N} \sum_{k=1}^{N} \lambda_{j}\left(\lambda_{j}-\lambda_{k}\right)^{2}= \\
=(N-1) \sum_{j=1}^{N} \lambda_{j}^{3}-\sum_{j=1}^{N} \sum_{k \neq j ; k=1}^{N} \lambda_{j}^{2} \lambda_{k} \\
0 \leq \Omega \triangleq \sum_{j=1}^{N} \sum_{k \neq j ; k=1}^{N-1} \sum_{\ell \neq j, k ; \ell=2}^{N} \lambda_{j}\left(\lambda_{k}-\lambda_{\ell}\right)^{2}= \\
=(N-2) \sum_{j=1}^{N} \sum_{k \neq j ; k=1}^{N} \lambda_{j}^{2} \lambda_{k} \\
-6 \sum_{j=1}^{N-2} \sum_{k=j+1}^{N-1} \sum_{\ell=k+1}^{N} \lambda_{j} \lambda_{k} \lambda_{\ell}
\end{gathered}
$$

e compute $(N-1) \Theta+\Omega \geq 0$, que se escreve

$$
\begin{aligned}
(N-1)^{2} \sum_{j=1}^{N} \lambda_{j}^{3}-\sum_{j=1}^{N} \sum_{k \neq j ; k=1}^{N} \lambda_{j}^{2} \lambda_{k} & \\
& -6 \sum_{j=1}^{N-2} \sum_{k=j+1}^{N-1} \sum_{\ell=k+1}^{N} \lambda_{j} \lambda_{k} \lambda_{\ell} \geq 0
\end{aligned}
$$


implicando que o termo dentro dos parênteses em (33) é sempre positivo, e que (21) é válida e, portanto, o teorema está provado.

Assim como no caso contínuo, pode-se impor sem perda de generalidade que o lado esquerdo de (28) seja menor que -I (condição necessária para a estabilidade, que define uma pequena folga para os termos cruzados $j, k$ e $\ell$, explorada pelo teorema). Note também que o resultado do Teorema 2 engloba a estabilidade quadrática como um caso particular, pois se existe $P=P^{*}=P_{1}=P_{2}=\cdots=P_{N}>0$ satisfazendo (28), então (29) e (30) são sempre satisfeitas. Para $N=1$, apenas (28) precisa ser verificada, recaindo-se no resultado do Lema 1 e, obviamente, a condição (30) só precisa ser considerada nos casos em que $N \geq 3$. Note que, no caso discreto, houve um aumento da complexidade em relação ao resultado do Teorema 1 , pois apesar do número de variáveis escalares ter se mantido igual a $N d n(d n+1) / 2$, o número de LMIs agora é dado por $\left(N^{3}+3 N^{2}+8 N\right) / 6$. Finalmente, observe que no caso de matrizes polinomiais na forma $A_{j}(s)=A_{j}-s \mathbf{I}_{n}$, as condições do Teorema 2 se reduzem ao resultado de estabilidade robusta de sistemas discretos (Ramos e Peres, 2001a), seguindo desenvolvimento similar ao apresentado para o caso contínuo (veja os comentários após a prova do Teorema 1).

Apesar de apenas o semi-plano esquerdo e o círculo unitário centrado na origem terem sido investigados neste trabalho, outras regiões de estabilidade poderiam ser analisadas, resultando em extensões dos teoremas 1 e 2 para diferentes matrizes $B$. Por exemplo, a $\mathcal{D}$-estabilidade de politopos de matrizes polinomiais em relação a um círculo de raio $r$, com centro em $(-d-r, 0)$, deslocado de uma distância $d$ à esquerda do eixo imaginário, como descrito em Haddad e Bernstein (1992), poderia ser investigada através das condições do Teorema 2 , trocando-se $B_{d}$ por

$$
B_{r d}=\left[\begin{array}{cc}
2 d+d^{2} / r & 1+d / r \\
1+d / r & 1 / r
\end{array}\right]
$$

O uso de matrizes $P(\lambda)$ dependentes de parâmetros, dadas por (17), permitiu o estabelecimento de condições suficientes para a estabilidade robusta do politopo de matrizes polinomiais $\mathfrak{P}$. Usando uma extensão do resultado apresentado em Leite e Peres (2003), condições ainda menos conservadoras podem ser obtidas.

LEMA 4 O politopo de matrizes polinomiais $\mathfrak{P}$ é robustamente estável se e somente existirem matrizes $P(\lambda) \in$ $\mathbb{C}^{d n \times d n}, j=1, \ldots, N$ e $Q(\lambda) \in \mathbb{C}^{2 d n \times n}$ solução do problema de factibilidade das LMIs

$$
\begin{gathered}
{\left[\begin{array}{c}
\mathcal{R} \\
\mathcal{A}(\lambda)
\end{array}\right]^{*}\left[\begin{array}{cc}
B \otimes P(\lambda) & Q(\lambda) \\
Q(\lambda)^{*} & 0
\end{array}\right]\left[\begin{array}{c}
\mathcal{R} \\
\mathcal{A}(\lambda)
\end{array}\right]<0} \\
P(\lambda)=P(\lambda)^{*}>0
\end{gathered}
$$

Prova: Primeiramente, a suficiência. Para $\lambda$ fixo, a condição do Lema 4 pode ser reescrita como

$$
\mathcal{R}^{*}(B \otimes P(\lambda)) \mathcal{R}+\mathcal{A}(\lambda)^{*} Q(\lambda)^{*} \mathcal{R}+\mathcal{R}^{*} Q(\lambda) \mathcal{A}(\lambda)<0
$$

Multiplicando-se à esquerda por $\mathcal{N}_{\mathcal{A}(\lambda)}^{*}$ e à direita por $\mathcal{N}_{\mathcal{A}(\lambda)}$, tem-se (18), que garante (pelo Lema 1) que a matriz polinomial $A(s, \lambda)$ dada em (2) é estável para todo $\lambda$ admissível e, portanto, o politopo $\mathfrak{P}$ é estável. A necessidade pode ser mostrada pelo Lema de Finsler (de Oliveira e Skelton, 2001). Supondo que (18) é verificada para todo $\lambda$ admissível, então existe $\mu(\lambda) \in \mathbb{R}$ tal que $\mathcal{R}^{*}(B \otimes P(\lambda)) \mathcal{R}-\mu(\lambda) \mathcal{A}(\lambda)^{*} \mathcal{A}(\lambda)<$ 0 , que é obtido em (38) com a escolha particular $\mathcal{R}^{*} Q(\lambda)=$ $-\mu(\lambda) \mathcal{A}(\lambda)^{*} / 2$.

Tal como foi formulado, o Lema 4 é de pouca utilidade numérica, pois o conjunto $\mathfrak{P}$ teria que ser testado para todo $\lambda$. O teorema a seguir fornece condições suficientes para a estabilidade robusta de $\mathfrak{P}$ em relação a uma região genérica do plano complexo definida pela matriz $B$, a partir de um número finito de desigualdades matriciais lineares que usam somente as informações dos vértices.

TEOREMA 3 O politopo de matrizes polinomiais $\mathfrak{P}$ é robustamente estável se existirem matrizes $P_{j} \in \mathbb{C}^{d n \times d n}$ e matrizes $Q_{j} \in \mathbb{C}^{d n \times n}, j=1, \ldots, N$, soluções do problema de factibilidade das LMIs

$$
\begin{aligned}
& \mathcal{R}^{*}\left(B \otimes P_{j}\right) \mathcal{R}+\mathcal{A}_{j}^{*} Q_{j}^{*} \mathcal{R}+\mathcal{R}^{*} Q_{j} \mathcal{A}_{j}<-\mathbf{I} \\
& j=1, \ldots, N \\
& \mathcal{R}^{*}\left[B \otimes\left(2 P_{j}+P_{k}\right)\right] \mathcal{R}+\left(\mathcal{A}_{j}^{*} Q_{j}^{*}+\mathcal{A}_{j}^{*} Q_{k}^{*}+\mathcal{A}_{k}^{*} Q_{j}^{*}\right) \mathcal{R} \\
& +\mathcal{R}^{*}\left(Q_{j} \mathcal{A}_{j}+Q_{k} \mathcal{A}_{j}+Q_{j} \mathcal{A}_{k}\right)<\frac{1}{(N-1)^{2}} \mathbf{I} ; \\
& j=1, \ldots, N, k \neq j, k=1, \ldots, N \\
& 2 \mathcal{R}^{*}\left[B \otimes\left(P_{j}+P_{k}+P_{\ell}\right)\right] \mathcal{R} \\
& +\left(\mathcal{A}_{j}^{*} Q_{\ell}^{*}+\mathcal{A}_{\ell}^{*} Q_{j}^{*}+\mathcal{A}_{k}^{*} Q_{\ell}^{*}+\mathcal{A}_{\ell}^{*} Q_{k}^{*}+\mathcal{A}_{j}^{*} Q_{k}^{*}+\mathcal{A}_{k}^{*} Q_{j}^{*}\right) \mathcal{R} \\
& +\mathcal{R}^{*}\left(Q_{j} \mathcal{A}_{\ell}+Q_{\ell} \mathcal{A}_{j}+Q_{k} \mathcal{A}_{\ell}+Q_{\ell} \mathcal{A}_{k}+Q_{k} \mathcal{A}_{j}+Q_{j} \mathcal{A}_{k}\right) \\
& \begin{array}{c}
<\frac{6}{(N-1)^{2}} \mathbf{I} \quad j=1, \ldots, N-2, k=j+1, \ldots, N-1 \\
\ell=k+1, \ldots, N
\end{array} \\
& P_{j}=P_{j}^{*}>0 \quad ; j=1, \ldots, N
\end{aligned}
$$

com $\mathcal{N}_{\mathcal{A}_{j}}$ e $\mathcal{R}$ dados, respectivamente, em (7) e (10). No caso afirmativo, $P(\lambda)$ dada por (17) é definida positiva e satisfaz (36) com

$$
Q(\lambda)=\sum_{j=1}^{N} \lambda_{j} Q_{j}>0, \lambda_{j} \geq 0, \sum_{j=1}^{N} \lambda_{j}=1
$$

para todo $\mathcal{A}(\lambda)$. 
Prova: Segue passos similares aos da prova do Teorema 2 (caso discreto). O lado esquerdo da equação (36) (ou, equivalentemente, de (38)) pode ser escrito como uma soma ponderada por coeficientes positivos dos termos que aparecem no lado esquerdo das desigualdades do Teorema 3. Impondo-se as condições (39)-(42) tem-se que $P(\lambda)=P(\lambda)^{*}>0$ e $Q(\lambda)$ dado por (43) verificam as condições do Lema 4 , garantindo a estabilidade do politopo $\mathfrak{P}$.

O Teorema 3 contém os resultados anteriores como casos particulares e fornece avaliações bem menos conservadoras da estabilidade robusta. Fazendo $Q_{j}=Q, j=1, \ldots, N$, do teorema recuperam-se as condições do Lema 2. As condições do Teorema 1 são recuperadas pré-multiplicando (39), (40) e (41) respectivamente por

$$
\mathcal{N}_{\mathcal{A}_{j}}^{*}, \mathcal{N}_{2}^{*} \mathcal{A}_{j}+\mathcal{A}_{k} \text { e } \mathcal{N}_{2}^{*}\left(\mathcal{A}_{j}+\mathcal{A}_{k}+\mathcal{A}_{\ell}\right)
$$

Mais precisamente, recupera-se uma formulação mais geral do que a do Teorema 1 , que pode ser obtida a partir da equação (25) multiplicada por $\sum_{j=1}^{N} \lambda_{j}=1$. As condições do Teorema 2 são recuperadas de forma similar. $\mathrm{O}$ número de LMIs do Teorema 3 é o mesmo do Teorema 2, mas o número de variáveis escalares é maior, dado por $K_{T 3}=$ $N d n(d n+2 n-1) / 2$.

\section{EXEMPLOS}

Embora a formulação teórica contemple polinômios matriciais com coeficientes complexos, nesta seção apenas o caso real é considerado. Uma comparação numérica utilizando-se valores de $n \in[1,4], d \in[2,4]$ e $N \in[2,5]$ foi realizada para os casos contínuo e discreto no tempo. Para cada situação 100 politopos de matrizes polinomiais foram gerados (no caso escalar, isto é, $n=1$, foram 1000 politopos de polinômios para $d \in[2,7]$ e $N \in[2,7]$ ) adotando-se o seguinte procedimento: i) Os vértices do politopo são gerados com matrizes cujos elementos são números reais uniformemente distribuídos no intervalo $[-1,1]$; ii) Para cada vértice, é garantido inicialmente pelo menos um zero com parte real no intervalo $\left[-5 \times 10^{-2},-1 \times 10^{-5}\right]$ (caso contínuo) ou valor absoluto no intervalo $[0.95,0.98]$ (caso discreto). iii) Os zeros dos politopos são calculados através de uma varredura fina e determina-se o zero com maior parte real $\sigma_{\max }$ (caso contínuo) ou maior valor absoluto $r_{\max }$ (caso discreto). Em seguida todo o politopo é deslocado de maneira a garantir que exista pelo menos um zero com parte real no intervalo $\left[-0.001,-1 \times 10^{-5}\right]$ (caso contínuo) ou com valor absoluto no intervalo $[0.98,0.999]$ (caso discreto). O deslocamento é feito substituindo-se $s$ por $s+\sigma_{\max }+\varepsilon$ (caso contínuo) ou $s$ por $s /\left(r_{\max } / \varepsilon\right)$ (caso discreto).

As tabelas 1 e 3 mostram os resultados para o caso contínuo. A condição do Lema 3 (L3) é sem dúvida a mais conservadora (de fato, o Lema 2 e o Teorema 1 englobam os resul- tados de L3). Em geral, o Teorema 1 (T1) fornece resultados menos conservadores do que o Lema 2 (L2). Embora L2 forneça resultados um pouco melhores do que T1 para casos de dimensões pequenas, com o aumento da complexidade essa tendência se inverte. Para o caso escalar $(n=1)$, T1 identifica mais politopos estáveis do que L2 em 18 dos 36 casos (12 empates), com percentagens relativas de $0.1 \%$ a $22.7 \%$ (média de $5.0 \%$ ). Quando L2 ganha (somente 6 casos) as percentagens vão de $0.5 \%$ a $6.8 \%$ (média de $3.1 \%$ ). Para $n=[2,4]$, L2 identifica mais politopos estáveis somente em 3 casos, com percentagens relativas de $1.0 \%$ a $4.0 \%$ com média de $2.8 \%$. T1 ganha em 26 casos ( 7 empates), com percentagens relativas de $2.1 \%$ a $72.4 \%$ (média de $35.1 \%$ ). Teorema 3 (T3) contém os demais resultados como casos particulares, fornecendo avaliações positivas sempre que um dos métodos obtém sucesso, apresentando um desempenho bem próximo da necessidade. De maneira geral, T3 reconhece mais de $95 \%$ dos politopos estáveis, mantendo esse patamar mesmo quando as dimensões do problema crescem.

Os resultados para o caso discreto são similares, como mostrado nas tabelas 2 e 4 . Novamente, L3 constitui-se no teste mais conservador. L2 fornece resultados levemente melhores no caso escalar $(n=1)$, identificando mais politopos estáveis em 14 dos 36 experimentos (11 empates), com percentagens relativas de $0.1 \%$ a $5.1 \%$ (média de $1.2 \%$ ). O Teorema 2 (T2) acerta em 11 casos, com percentagens relativas de $0.1 \%$ a $2.2 \%$ (média de $0.9 \%$ ). T2 torna-se mais efetivo nas avaliações quando a complexidade dos testes aumenta, vencendo, para $n=[2,4]$, em 23 casos dos 36 pesquisados (2 empates), com percentagens relativas de $1.0 \%$ a $34.2 \%$ (média de 10.2\%). Já L2 fornece resultados positivos em 11 casos, com percentagens relativas de $1.0 \%$ a $5.1 \%$ (média de $2.2 \%$ ). Como no caso contínuo, o desempenho de T3 é bem superior ao dos demais métodos.

Alguns exemplos particulares foram também investigados. No primeiro, foi gerado um polinômio escalar $(n=1)$ com coeficientes confinados a determinados intervalos. O objetivo deste exemplo é mostrar que com o aumento da complexidade do problema (associada ao aumento do número de vértices do politopo), a condição apresentada em L2 pode falhar, enquanto que T1 (e também T3) confirmam a estabilidade do politopo. O politopo de polinômios escalares sob análise é descrito como

$$
a(s)=\alpha_{0}+\alpha_{1} s+\alpha_{2} s^{2}+\alpha_{3} s^{3}+s^{4}
$$

com coeficientes $\alpha_{i}, i=0, \ldots, 3$, pertencendo ao hipercubo

$$
\begin{aligned}
& \left(\alpha_{0}, \alpha_{1}, \alpha_{2}, \alpha_{3}\right) \in([0.0683,3500.4237] ;[683.9193, \\
& 1476.1494] ;[220.2668,279.9684] ;[25.024,26.5218])
\end{aligned}
$$

Usando-se o resultado de Kharitonov (Kharitonov, 1978), sabe-se que para concluir sobre a estabilidade do politopo 
Tabela 1: Politopos estáveis identificados para $n=1, d=[2,7]$ (caso contínuo).

\begin{tabular}{|c|c|c|c|c|c|c|c|c|c|c|c|c|}
\hline \multirow{2}{*}{$N^{d}$} & \multicolumn{4}{|c|}{2} & \multicolumn{4}{|c|}{3} & \multicolumn{4}{|c|}{4} \\
\hline & L3 & L2 & $\mathrm{T} 1$ & T3 & L3 & L2 & $\mathrm{T} 1$ & T3 & L3 & L2 & $\mathrm{T} 1$ & T3 \\
\hline 2 & 331 & 1000 & 1000 & 1000 & 367 & 1000 & 1000 & 1000 & 349 & 1000 & 1000 & 1000 \\
\hline 3 & 185 & 1000 & 1000 & 1000 & 184 & 997 & 990 & 1000 & 180 & 1000 & 1000 & 1000 \\
\hline 4 & 87 & 1000 & 1000 & 1000 & 90 & 991 & 967 & 996 & 79 & 998 & 993 & 998 \\
\hline 5 & 57 & 1000 & 1000 & 1000 & 51 & 990 & 958 & 995 & 41 & 992 & 995 & 999 \\
\hline 6 & 24 & 1000 & 1000 & 1000 & 33 & 980 & 932 & 996 & 19 & 979 & 987 & 997 \\
\hline 7 & 17 & 1000 & 1000 & 1000 & 20 & 969 & 903 & 991 & 7 & 970 & 978 & 998 \\
\hline$d$ & & \multicolumn{4}{|c|}{0} & \multicolumn{4}{|c|}{ t } \\
\hline$N$ & L3 & L2 & $\mathrm{T} 1$ & T3 & L3 & L2 & $\mathrm{T} 1$ & T3 & L3 & L2 & $\mathrm{T} 1$ & $\mathrm{~T} 3$ \\
\hline 2 & 327 & 1000 & 1000 & 1000 & 322 & 1000 & 1000 & 1000 & 296 & 998 & 1000 & 1000 \\
\hline 3 & 146 & 1000 & 1000 & 1000 & 114 & 998 & 999 & 999 & 111 & 987 & 998 & 999 \\
\hline 4 & 50 & 998 & 1000 & 1000 & 47 & 984 & 998 & 999 & 24 & 958 & 999 & 1000 \\
\hline 5 & 14 & 990 & 997 & 997 & 24 & 965 & 999 & 999 & 15 & 915 & 998 & 1000 \\
\hline 6 & 13 & 974 & 996 & 996 & 11 & 915 & 998 & 998 & 5 & 855 & 999 & 999 \\
\hline 7 & 7 & 925 & 993 & 997 & 4 & 860 & 996 & 996 & 4 & 772 & 999 & 999 \\
\hline
\end{tabular}

Tabela 2: Politopos estáveis identificados para $n=1, d=[2,7]$ (caso discreto).

\begin{tabular}{|c|c|c|c|c|c|c|c|c|c|c|c|c|}
\hline \multirow{2}{*}{$N^{d}$} & \multicolumn{4}{|c|}{2} & \multicolumn{4}{|c|}{3} & \multicolumn{4}{|c|}{4} \\
\hline & L3 & L2 & $\mathrm{T} 2$ & T3 & L3 & L2 & $\mathrm{T} 2$ & T3 & L3 & L2 & $\mathrm{T} 2$ & T3 \\
\hline 2 & 364 & 1000 & 1000 & 1000 & 344 & 1000 & 1000 & 1000 & 283 & 1000 & 1000 & 1000 \\
\hline 3 & 105 & 1000 & 991 & 1000 & 55 & 1000 & 994 & 1000 & 27 & 1000 & 998 & 1000 \\
\hline 4 & 21 & 1000 & 985 & 1000 & 8 & 993 & 986 & 998 & 2 & 995 & 995 & 999 \\
\hline 5 & 8 & 1000 & 981 & 1000 & 3 & 971 & 983 & 998 & 0 & 992 & 989 & 1000 \\
\hline 6 & 2 & 1000 & 959 & 1000 & ( & 950 & 969 & 998 & 0 & 975 & 985 & 1000 \\
\hline 7 & 0 & 1000 & 949 & 1000 & & 925 & 946 & 990 & & 973 & 981 & 999 \\
\hline$d$ & & & \multicolumn{4}{|c|}{1} \\
\hline$N$ & L3 & L2 & $\mathrm{T} 2$ & T3 & L3 & L2 & $\mathrm{T} 2$ & T3 & L3 & L2 & $\mathrm{T} 2$ & T3 \\
\hline 2 & 262 & 1000 & 1000 & 1000 & 284 & 1000 & 1000 & 1000 & 254 & 1000 & 1000 & 1000 \\
\hline 3 & 33 & 1000 & 997 & 1000 & 24 & 1000 & 1000 & 1000 & 23 & 1000 & 1000 & 1000 \\
\hline 4 & 2 & 999 & 998 & 1000 & 3 & 1000 & 999 & 1000 & 1 & 1000 & 1000 & 1000 \\
\hline 5 & 1 & 993 & 991 & 1000 & 0 & 999 & 998 & 1000 & 0 & 1000 & 1000 & 1000 \\
\hline 6 & 0 & 988 & 993 & 1000 & 0 & 997 & 998 & 1000 & 0 & 996 & 997 & 1000 \\
\hline 7 & 0 & 987 & 993 & 1000 & 0 & 994 & 998 & 1000 & 0 & 994 & 998 & 1000 \\
\hline
\end{tabular}

Tabela 3: Politopos estáveis identificados para $n=[2,4]$ e $d=[2,4]$ (caso contínuo).

\begin{tabular}{|c|c|c|c|c|c|c|c|c|c|c|c|c|c|}
\hline \multirow[b]{2}{*}{$n$} & \multirow{2}{*}{${ }_{N}{ }^{d}$} & \multicolumn{4}{|c|}{2} & \multicolumn{4}{|c|}{3} & \multicolumn{4}{|c|}{4} \\
\hline & & L3 & L2 & T1 & T3 & L3 & L2 & T1 & T3 & L3 & L2 & T1 & T3 \\
\hline & 2 & 28 & 10 & 96 & 100 & 23 & 98 & 97 & 100 & 20 & 98 & 98 & 99 \\
\hline$?$ & 3 & 4 & 89 & 86 & 97 & 2 & 92 & 94 & 98 & 1 & 93 & 95 & 99 \\
\hline 2 & 4 & 2 & 76 & 90 & 94 & 1 & 80 & 89 & 96 & $\overline{0}$ & 78 & 96 & 96 \\
\hline & 5 & 1 & 64 & 88 & 97 & 1 & 72 & 93 & 96 & 0 & 65 & 93 & 93 \\
\hline \multirow{4}{*}{3} & 2 & 23 & 100 & 100 & 100 & 14 & 99 & 99 & 99 & 15 & 98 & 98 & 98 \\
\hline & 3 & 2 & 80 & 92 & 97 & 2 & 81 & 96 & 96 & 1 & 84 & 97 & 98 \\
\hline & 4 & 2 & 48 & 82 & 95 & 0 & 48 & 91 & 94 & 0 & 51 & 96 & 96 \\
\hline & 5 & 2 & 40 & 90 & 90 & 1 & 40 & 87 & 90 & 1 & 44 & 95 & 95 \\
\hline \multirow{4}{*}{4} & 2 & 27 & 96 & 96 & 99 & 14 & 97 & 97 & 99 & 16 & 98 & 98 & 98 \\
\hline & 3 & 1 & 67 & 88 & 94 & 1 & 77 & 97 & 98 & 2 & 65 & 96 & 97 \\
\hline & 4 & 0 & 43 & 86 & 90 & 0 & 37 & 92 & 98 & 0 & 50 & 94 & 95 \\
\hline & 5 & 1 & 25 & 81 & 91 & 0 & 24 & 87 & 98 & 0 & 28 & 89 & 89 \\
\hline
\end{tabular}


Tabela 4: Politopos estáveis identificados para $n=[2,4]$ e $d=[2,4]$ (caso discreto).

\begin{tabular}{|c|c|c|c|c|c|c|c|c|c|c|c|c|c|}
\hline$n$ & $d$ & \multicolumn{9}{|c|}{$\mathbf{2}$} & \multicolumn{6}{|c|}{$\mathbf{3}$} & \multicolumn{4}{|c|}{$\mathbf{4}$} \\
\hline & $N$ & L3 & L2 & T1 & T3 & L3 & L2 & T1 & T3 & L3 & L2 & T1 & T3 \\
\hline \multirow{4}{*}{2} & 2 & 16 & 99 & 94 & 99 & 13 & 99 & 99 & 99 & 8 & 100 & 99 & 100 \\
\hline & 3 & 1 & 92 & 88 & 98 & 0 & 97 & 95 & 98 & 0 & 98 & 98 & 99 \\
& 4 & 0 & 80 & 81 & 94 & 0 & 90 & 92 & 96 & 0 & 93 & 96 & 99 \\
& 5 & 0 & 70 & 75 & 97 & 0 & 81 & 88 & 96 & 0 & 88 & 95 & 95 \\
\hline \multirow{4}{*}{$\mathbf{3}$} & 2 & 9 & 99 & 95 & 99 & 7 & 99 & 98 & 100 & 5 & 100 & 99 & 100 \\
& 3 & 1 & 88 & 87 & 98 & 0 & 95 & 96 & 98 & 0 & 97 & 98 & 99 \\
& 4 & 0 & 74 & 83 & 95 & 0 & 84 & 92 & 93 & 0 & 87 & 99 & 99 \\
& 5 & 0 & 58 & 74 & 93 & 0 & 72 & 90 & 96 & 0 & 80 & 97 & 97 \\
\hline \multirow{4}{*}{4} & 2 & 5 & 99 & 96 & 99 & 4 & 100 & 99 & 100 & 4 & 100 & 99 & 100 \\
\hline & 3 & 0 & 87 & 90 & 100 & 0 & 94 & 97 & 98 & 0 & 97 & 100 & 100 \\
\hline & 4 & 0 & 72 & 83 & 95 & 0 & 87 & 92 & 99 & 0 & 89 & 99 & 100 \\
\hline & 5 & 0 & 52 & 79 & 94 & 0 & 73 & 92 & 96 & 0 & 79 & 98 & 99 \\
\hline
\end{tabular}

considerado é necessário e suficiente testar apenas os quatro polinômios

$$
\begin{aligned}
& a_{1}(s)=0.0683+683.9193 s+279.9684 s^{2}+26.5218 s^{3}+s^{4} \\
& a_{2}(s)=3500.4237+1476.1494 s+220.2668 s^{2}+25.024 s^{3}+s^{4} \\
& a_{3}(s)=3500.4237+683.9193 s \\
&+ 220.2668 s^{2}+26.5218 s^{3}+s^{4} \\
& a_{4}(s)=0.0683+1476.1494 s+279.9684 s^{2}+25.024 s^{3}+s^{4}
\end{aligned}
$$

Tanto L2 quanto T1 e T3 concluem positivamente sobre a estabilidade do politopo formado pelos polinômios $\left\{a_{1}(s), a_{2}(s), a_{3}(s), a_{4}(s)\right\}$. Entretanto, L2 falha ao serem considerados todos os $N=16$ vértices existentes no problema, o que não ocorre com T1 e T3.

Como segundo exemplo, foi considerado um politopo de matrizes polinomiais, caso contínuo no tempo, de dimensão $n=2$, grau $d=2$ e vértices $N=3$ dados por

$$
\begin{aligned}
A_{1} & =\left[\begin{array}{cccccc}
0.73 & -0.14 & 0.69 & -0.60 & 1 & 0 \\
-0.98 & 0.94 & -0.08 & 1.09 & 0 & 1
\end{array}\right] \\
A_{2} & =\left[\begin{array}{cccccc}
0.62 & 0.56 & 0.37 & 0.74 & 1 & 0 \\
0.27 & 0.73 & -0.17 & 0.15 & 0 & 1
\end{array}\right] \\
A_{3} & =\left[\begin{array}{cccccc}
0.86 & -0.12 & 0.18 & -0.07 & 1 & 0 \\
0.36 & 0.26 & 0.84 & 0.80 & 0 & 1
\end{array}\right]
\end{aligned}
$$

Os testes baseados na estabilidade quadrática (L3) e na estabilidade robusta de L2 falharam, mas T1 e T3 obtiveram soluções factíveis, garantindo a estabilidade robusta do politopo, como pode ser verificado na figura 1 .

Para o terceiro exemplo, como ilustração do caso discreto, foi gerado um politopo de matrizes polinomiais $\operatorname{com} n=2$,

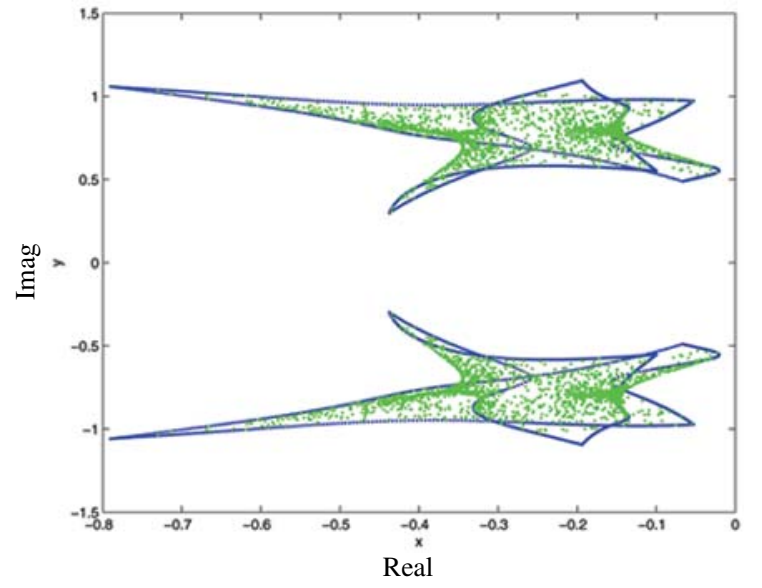

Figura 1: Lugar das raízes para politopo de matrizes polinomiais descritas pelos vértices (44)-(46) (caso contínuo) identificado como estável apenas pelos Teoremas 1 e 3 .

$d=2$ e $N=3$ vértices dados por

$$
\begin{gathered}
A_{1}=\left[\begin{array}{cccccc}
0.96 & 0.76 & 0.90 & 0.21 & 1 & 0 \\
0.34 & 0.65 & -0.43 & -0.42 & 0 & 1
\end{array}\right] \\
A_{2}=\left[\begin{array}{cccccc}
-0.60 & -0.52 & -0.01 & 0.30 & 1 & 0 \\
-0.65 & 0.59 & 0.31 & -0.79 & 0 & 1
\end{array}\right] \\
A_{3}=\left[\begin{array}{cccccc}
-0.18 & -0.62 & -0.83 & -0.41 & 1 & 0 \\
0.35 & -0.42 & 0.21 & 0.29 & 0 & 1
\end{array}\right]
\end{gathered}
$$

$\mathrm{O}$ lugar das raízes está mostrado na figura 2 . Também nesse caso, falharam os testes de L2 e L3, e apenas T2 e T3 forneceram solução factível.

É importante ressaltar que, embora as condições L2 e as propostas neste trabalho (T1, T2 e T3) contenham o teste de estabilidade quadrática (L3) como um caso particular, L2 e T1/T2 são condições suficientes independentes, ambas contidas na condição T3. Em outras palavras, podem existir politopos estáveis identificados por T1/T2 mas não por L2 e 


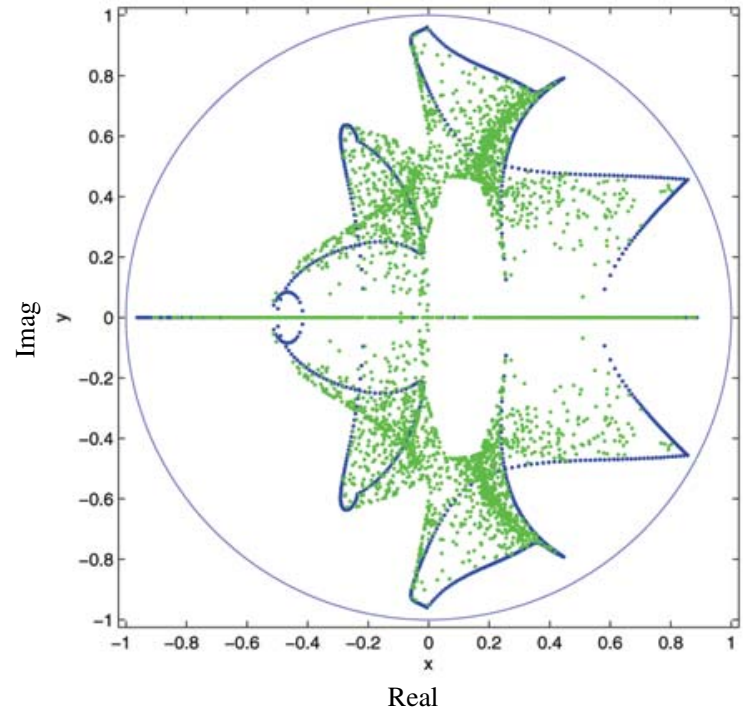

Figura 2: Lugar das raízes para politopo de matrizes polinomiais descritas pelos vértices (44)-(46) (caso contínuo) identificado como estável apenas pelos Teoremas 1 e 3.

vice-versa. A análise numérica apresentada mostra que, com o aumento na complexidade dos testes ( $n, d$ e $N$ crescentes), as condições T1/T2 reconhecem mais politopos estáveis do que L2.

As condições mais abrangentes do Teorema 3 (T3) forneceram avaliações positivas sempre que um dos testes foi positivo, identificando inúmeros politopos de matrizes polinomiais estáveis em situações nas quais os demais métodos falharam. Por exemplo, o polinômio de matrizes polinomiais (sistemas contínuos, $n=2, d=2$ e $N=3$ vértices) dado abaixo é estável, como pode ser visto na Figura 3, porém apenas o Teorema 3 conseguiu a identificação correta (os demais testes falharam).

$$
\begin{aligned}
A_{1} & =\left[\begin{array}{cccccc}
0.99 & -1.03 & 0.82 & -0.79 & 1 & 0 \\
0.67 & 0.13 & 0.00 & 1.05 & 0 & 1
\end{array}\right] \\
A_{2} & =\left[\begin{array}{cccccc}
0.85 & -0.49 & 0.59 & -0.05 & 1 & 0 \\
0.75 & -0.33 & 0.75 & 0.26 & 0 & 1
\end{array}\right] \\
A_{3} & =\left[\begin{array}{cccccc}
0.14 & 1.25 & 0.67 & 0.67 & 1 & 0 \\
-0.68 & 1.13 & 0.01 & 1.79 & 0 & 1
\end{array}\right]
\end{aligned}
$$

De maneira similar, o politopo de matrizes polinomiais descrito pelas matrizes abaixo (caso discreto) apenas foi identificado como estável (veja a Figura 4) pelo Teorema 3. Os demais testes nada concluíram.

$$
A_{1}=\left[\begin{array}{cccccc}
0.25 & 0.93 & -0.95 & -0.60 & 1 & 0 \\
-0.84 & 0.14 & 0.25 & 0.27 & 0 & 1
\end{array}\right]
$$

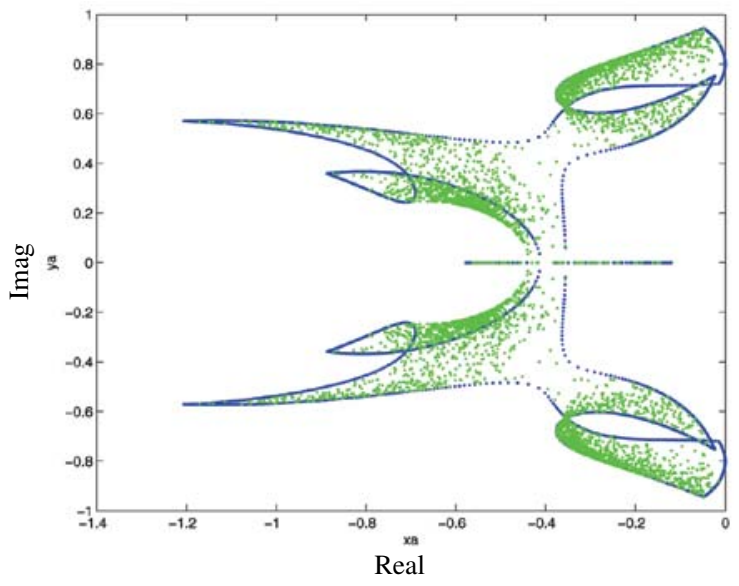

Figura 3: Lugar das raízes para politopo de matrizes polinomiais descritas pelos vértices (50)-(52) (caso contínuo) identificado como estável apenas pelo Teorema 3.

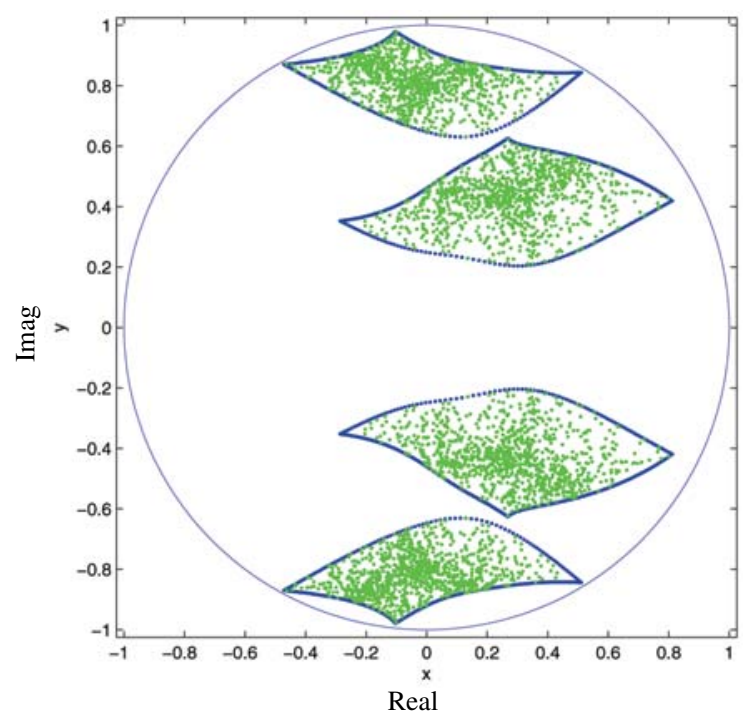

Figura 4: Lugar das raízes para politopo de matrizes polinomiais descritas pelos vértices (53)-(55) (caso discreto) identificado como estável apenas pelo Teorema 3.

$$
\begin{aligned}
& A_{2}=\left[\begin{array}{cccccc}
0.11 & -0.47 & -0.26 & -0.67 & 1 & 0 \\
0.38 & 0.20 & 0.35 & -0.19 & 0 & 1
\end{array}\right] \\
& A_{3}=\left[\begin{array}{cccccc}
0.93 & 0.42 & 0.00 & -0.93 & 1 & 0 \\
-0.14 & 0.42 & -0.03 & -0.33 & 0 & 1
\end{array}\right]
\end{aligned}
$$

Os testes foram realizados em um Pentium IV $2.6 \mathrm{GHz}$ com 512 Mbytes de memória RAM usando MATLAB e o pacote LMI Control Toolbox (Gahinet et al., 1995). O tempo médio de CPU gasto em cada teste de politopo é mostrado na Tabela 5 para sistemas contínuos (esquerda) e para sistemas dis- 
Tabela 5: Tempo médio (segundos) para avaliação de um politopo estável para $n=[1,4], d=\{[5,7],[2,4]\}$ e $N=\{[5,7],[2,5]\}$ com L2, T1/T2 e T3 (caso contínuo à esquerda, caso discreto à direita). O símbolo “-" indica tempos menores do que 0.1 segundos.

\begin{tabular}{|c|c|c|c|c|c|c|c|c|c|c|}
\hline \multirow{2}{*}{$n$} & \multirow{2}{*}{$\begin{array}{c}{ }_{N}^{d} \\
\end{array}$} & \multicolumn{3}{|c|}{5} & \multicolumn{3}{|c|}{6} & \multicolumn{3}{|c|}{7} \\
\hline & & L2 & T1 & T3 & L2 & T1 & T3 & L2 & T1 & T3 \\
\hline \multirow{3}{*}{1} & 5 & 0.2 & 0.2 & 2.2 & 0.5 & 0.4 & 4.6 & 1.0 & 0.8 & 10.9 \\
\hline & 6 & 0.3 & 0.3 & 4.6 & 0.7 & 0.7 & 11.2 & 1.8 & 1.3 & 28.4 \\
\hline & 7 & 0.4 & 0.5 & 8.3 & 1.1 & 1.0 & 23.3 & 3.2 & 1.9 & 55.0 \\
\hline & & \multicolumn{3}{|c|}{2} & \multicolumn{3}{|c|}{3} & \multicolumn{3}{|c|}{4} \\
\hline \multirow{2}{*}{2} & 4 & - & - & 0.8 & 0.3 & 0.2 & 4.3 & 1.2 & 0.7 & 12.8 \\
\hline & 5 & 0.1 & 0.1 & 1.9 & 0.5 & 0.3 & 10.1 & 2.2 & 1.1 & 48.4 \\
\hline \multirow{4}{*}{3} & 2 & - & - & 0.4 & 0.6 & 0.2 & 1.9 & 2.3 & 0.4 & 5.8 \\
\hline & 3 & 0.3 & 0.1 & 1.9 & 1.4 & 0.4 & 10.0 & 4.9 & 1.4 & 35.2 \\
\hline & 4 & 0.5 & 0.2 & 6.6 & 3.0 & 0.8 & 30.9 & 17.2 & 3.5 & 140.0 \\
\hline & 5 & 0.6 & 0.3 & 15.3 & 5.0 & 1.4 & 121.9 & 23.9 & 8.3 & 395.5 \\
\hline \multirow{4}{*}{4} & 2 & 0.4 & - & 1.7 & 2.4 & 0.4 & 9.4 & 11.2 & 1.5 & 27.6 \\
\hline & 3 & 0.9 & 0.3 & 9.9 & 6.8 & 1.4 & 61.0 & 31.3 & 5.2 & 209.5 \\
\hline & 4 & 2.1 & 0.5 & 29.9 & 16.8 & 4.8 & 170.4 & 69.4 & 13.5 & 750.0 \\
\hline & 5 & 4.6 & 1.1 & 109.2 & 25.0 & 5.0 & 557.5 & 131.4 & 22.0 & 3179.3 \\
\hline
\end{tabular}

\begin{tabular}{|c|c|c|c|c|c|c|c|c|c|c|}
\hline \multirow{2}{*}{$n$} & \multirow{2}{*}{$N^{d}$} & \multicolumn{3}{|c|}{5} & \multicolumn{3}{|c|}{6} & \multicolumn{3}{|c|}{7} \\
\hline & & L2 & T2 & T3 & L2 & $\mathrm{T} 2$ & T3 & L2 & $\mathrm{T} 2$ & T3 \\
\hline \multirow{3}{*}{1} & 5 & 0.1 & 0.7 & 1.3 & 0.3 & 1.2 & 3.7 & 0.9 & 2.2 & 8.3 \\
\hline & 6 & 0.2 & 1.4 & 2.7 & 0.5 & 2.5 & 7.2 & 1.3 & 4.6 & 19.6 \\
\hline & 7 & 0.3 & 2.6 & 5.0 & 0.8 & 4.8 & 15.4 & 2.3 & 7.3 & 38.0 \\
\hline & & \multicolumn{3}{|c|}{2} & \multicolumn{3}{|c|}{3} & \multicolumn{3}{|c|}{4} \\
\hline \multirow{2}{*}{2} & 4 & - & 0.2 & 0.4 & 0.1 & 0.7 & 1.6 & 0.6 & 1.8 & 6.2 \\
\hline & 5 & - & 0.4 & 0.9 & 0.2 & 1.4 & 4.2 & 1.1 & 4.4 & 15.2 \\
\hline \multirow{4}{*}{3} & 2 & - & - & 0.2 & 0.2 & 0.3 & 0.8 & 1.3 & 0.7 & 3.7 \\
\hline & 3 & - & 0.3 & 0.8 & 0.6 & 1.0 & 4.7 & 3.1 & 3.4 & 17.7 \\
\hline & 4 & 0.2 & 0.9 & 2.8 & 1.2 & 3.2 & 15.1 & 6.5 & 9.1 & 55.5 \\
\hline & 5 & 0.2 & 1.7 & 6.8 & 2.1 & 8.1 & 36.5 & 12.5 & 23.6 & 152.6 \\
\hline \multirow{4}{*}{4} & 2 & 0.2 & 0.2 & 0.7 & 1.3 & 0.8 & 4.0 & 5.9 & 2.3 & 18.7 \\
\hline & 3 & 0.3 & 0.7 & 3.6 & 2.9 & 3.0 & 21.5 & 18.3 & 9.4 & 140.4 \\
\hline & 4 & 0.6 & 2.2 & 13.0 & 6.0 & 9.7 & 76.6 & 36.8 & 38.7 & 316.4 \\
\hline & 5 & 1.1 & 5.4 & 32.3 & 10.1 & 22.5 & 219.2 & 69.0 & 63.6 & 962.6 \\
\hline
\end{tabular}

cretos (direita), considerando somente as avaliações factíveis nas quais o tempo gasto foi maior ou igual a 0.1 segundos (“-” indica tempos menores do que 0.1 segundos). O tempo médio gasto pela condição L3, sempre inferior ao tempo dos demais testes, não é apresentado.

\section{CONCLUSÃO}

Neste trabalho, foram apresentadas novas condições suficientes para análise de estabilidade de politopos de matrizes polinomiais que se mostraram menos conservadoras que as existentes na literatura. A estabilidade robusta do politopo é verificada através de um teste de factibilidade de LMIs descritas apenas em função dos vértices do politopo, que pode ser realizado de maneira eficiente por métodos numéricos de complexidade polinomial.

\section{AGRADECIMENTOS}

Os autores agradecem o apoio das agências CAPES, CNPq, FAPEMIG e FAPESP, bem como os comentários e sugestões dos revisores.

\section{REFERÊNCIAS}

Ackermann, J. (1993). Robust Control: Systems with Uncertain Parameters, Springer Verlag, London, England.

Barmish, B. R. (1994). New Tools for Robustness of Linear Systems, Macmillan Publishing Company, New York, NY, USA.

Bartlett, A. C., Hollot, C. V. e Lin, H. (1988). Root locations of an entire polytope of polynomials: it suffices to

check the edges, Mathematics of Control, Signals and Systems 1: 61-71.

Bhattacharyya, S. P., Chapellat, H. e Keel, L. H. (1995). Robust Control: The Parametric Approach, Prentice-Hall Publishing Co., Upper Saddle River, NJ, USA.

Blondel, V. D. e Tsitsiklis, J. N. (2000). A survey of computational complexity results in systems and control, $\mathrm{Au}$ tomatica 36(9): 1249-1274.

Boyd, S., El Ghaoui, L., Feron, E. e Balakrishnan, V. (1994). Linear Matrix Inequalities in System and Control Theory, SIAM Studies in Applied Mathematics, Philadelphia, PA.

Chilali, M. e Gahinet, P. (1996). $\mathcal{H}_{\infty}$ design with pole placement constraints: an LMI approach, IEEE Transactions on Automatic Control 41(3): 358-367.

de Oliveira, M. C., Bernussou, J. e Geromel, J. C. (1999). A new discrete-time robust stability condition, Systems \& Control Letters 37(4): 261-265.

de Oliveira, M. C. e Skelton, R. E. (2001). Stability tests for constrained linear systems, in S. O. Reza Moheimani (ed.), Perspectives in Robust Control, Vol. 268 of Lecture Notes in Control and Information Science, Springer-Verlag, New York, pp. 241-257.

Gahinet, P., Nemirovski, A., Laub, A. J. e Chilali, M. (1995). LMI Control Toolbox User's Guide, The Math Works Inc., Natick, MA.

Geromel, J. C., de Oliveira, M. C. e Hsu, L. (1998). LMI characterization of structural and robust stability, Linear Algebra and Its Applications 285(1-3): 69-80. 
Geromel, J. C., Peres, P. L. D. e Bernussou, J. (1991). On a convex parameter space method for linear control design of uncertain systems, SIAM Journal on Control and Optimization 29(2): 381-402.

Gohberg, I., Lancaster, P. e Rodman, L. (1982). Matrix Polynomials, Academic Press, New York, NY, USA.

Haddad, W. M. e Bernstein, D. S. (1992). Controller design with regional pole constraints, IEEE Transactions on Automatic Control 37(1): 54-69.

Henrion, D., Arzelier, D., Peaucelle, D. e Šebek, M. (2001). An LMI condition for robust stability of polynomial matrix polytopes, Automatica 37(3): 461-468.

Henrion, D., Bachelier, O. e Šebek, M. (2001). D-stability of polynomial matrices, International Journal of Control 74(8): 845-846.

Kailath, T. (1980). Linear System, Prentice-Hall, Englewood Cliffs, NJ, USA.

Karl, W. C. e Verghese, G. C. (1993). A sufficient condition for the stability of interval matrix polynomials, IEEE Transactions on Automatic Control 38(7): 1139-1143.

Kharitonov, V. L. (1978). Asymptotic stability of an equilibrium position of a family of systems of linear differential equations, Differentsial'nye Uravneniya 14: 20862088.

Kučera, V. (1979). Discrete Linear Control: The Polynomial Equation Approach, Wiley, Chichester, England.

Leite, V. J. S., Montagner, V. F., de Oliveira, P. J., Oliveira, R. C. L. F., Ramos, D. C. W. e Peres, P. L. D. (2004). Estabilidade robusta de sistemas lineares através de desigualdades matriciais lineares, Revista Controle \& Automação da SBA 15(1): 24-40.

Leite, V. J. S. e Peres, P. L. D. (2003). An improved LMI condition for robust $\mathcal{D}$-stability of uncertain polytopic systems, IEEE Transactions on Automatic Control 48(3): 500-504.

Peaucelle, D., Arzelier, D., Bachelier, O. e Bernussou, J. (2000). A new robust $\mathcal{D}$-stability condition for real convex polytopic uncertainty, Systems \& Control Letters 40(1): 21-30.

Ramos, D. C. W. e Peres, P. L. D. (2001a). A less conservative LMI condition for the robust stability of discretetime uncertain systems, Systems \& Control Letters 43(5): 371-378.
Ramos, D. C. W. e Peres, P. L. D. (2001b). An LMI approach to compute robust stability domains for uncertain linear systems, Proceedings of the 2001 American Control Conference, Vol. 1, Arlington, VA, pp. 4073-4078.

Ramos, D. C. W. e Peres, P. L. D. (2002). An LMI condition for the robust stability of uncertain continuous-time linear systems, IEEE Transactions on Automatic Control 47(4): 675-678.

Willems, J. C. (1991). Paradigms and puzzles in the theory of dynamical systems, IEEE Transactions on Automatic Control 36(3): 259-294. 